\title{
Measuring image resolution in optical nanoscopy
}

\author{
Robert P J Nieuwenhuizen ${ }^{1}$, Keith A Lidke ${ }^{2}$, Mark Bates ${ }^{3}$, Daniela Leyton Puig ${ }^{4}$, David Grünwald ${ }^{5,6}$, \\ Sjoerd Stallinga ${ }^{1,7}$ \& Bernd Rieger ${ }^{1,7}$
}

\begin{abstract}
Resolution in optical nanoscopy (or super-resolution microscopy) depends on the localization uncertainty and density of single fluorescent labels and on the sample's spatial structure. Currently there is no integral, practical resolution measure that accounts for all factors. We introduce a measure based on Fourier ring correlation (FRC) that can be computed directly from an image. We demonstrate its validity and benefits on two-dimensional (2D) and 3D localization microscopy images of tubulin and actin filaments. Our FRC resolution method makes it possible to compare achieved resolutions in images taken with different nanoscopy methods, to optimize and rank different emitter localization and labeling strategies, to define a stopping criterion for data acquisition, to describe image anisotropy and heterogeneity, and even to estimate the average number of localizations per emitter. Our findings challenge the current focus on obtaining the best localization precision, showing instead how the best image resolution can be achieved as fast as possible.
\end{abstract}

The first and foremost law of conventional optical imaging science is that resolution is limited to a value on the order of $\lambda / \mathrm{NA}$, with $\lambda$ equal to the wavelength of light and NA to the numerical aperture of the imaging lens. Rayleigh and Sparrow captured this law through empirical resolution criteria. These criteria were placed on solid foundations by Abbe and Nyquist, who defined resolution as the inverse of the spatial bandwidth of the imaging system. This diffraction limit, however, can be overcome by numerous optical nanoscopy techniques, notably stimulated emission depletion $(\mathrm{STED})^{1}$, reversible saturable optical fluorescence transitions $(\text { RESOLFT })^{2}$, the family of localization microscopy techniques such as photoactivated localization microscopy (PALM), stochastic optical reconstruction microscopy (STORM), ground state depletion microscopy followed by individual molecule return (GSDIM) and direct STORM ${ }^{3-6}$ and statistical methods such as blinking fluorescence localization and super-resolution optical fluctuation imaging $(\mathrm{SOFI})^{7,8}$.

These revolutionary developments raise the question: what is resolution in diffraction-unlimited imaging? The resolving power of the instrument is often coupled to the uncertainty of localizing single emitters, that is, point sources. The closely related twopoint resolution can be given a precise meaning in the context of localization microscopy ${ }^{9}$, thus generalizing the Rayleigh criterion of conventional microscopy. These concepts characterize the resolution in images in which the structure of interest can be defined by a limited number of molecules-such as images of the nuclear pore complex ${ }^{10}$-or when investigating the relative position of different molecules ${ }^{11}$. However, if more-or-less continuous structures with a large number of potential labeling sites are imaged-for example, actin filaments or organelle membranesthen it is clear that the average density of localized fluorescent labels must also play a role. As early as the first demonstration of localization microscopy for cell imaging ${ }^{3}$, it was noted that "both parameters-localization precision and the density of rendered molecule-are key to defining performance...." The effects of labeling density and photoswitching kinetics on resolution have since been investigated experimentally ${ }^{12,13}$. Recently, an estimation-theoretic resolution concept was presented ${ }^{14}$ that combines both labeling density and localization uncertainty using an a priori model of the sample.

We conclude from all prior work that neither the average density of localized molecules needed for random Nyquist sampling nor the localization uncertainty alone is a suitable measure to characterize the resolution. In addition, the resolution depends on a multitude of other factors such as the link between the label and the structure, the underlying spatial structure of the sample itself and the extensive data processing required to produce a final super-resolution image comprising, for example, single-emitter candidate selection and localization algorithms. Ultimately, only an integral, image-based resolution measure not depending on any a priori information is suitable for determining what level of detail can be reliably discerned in a given image.

Here we propose an image-resolution measure that can be computed directly from experimental data alone. It is centered on the FRC (or, equivalently, the spectral signal-to-noise ratio), which is commonly used in the field of cryo-electron microscopy (cryoEM) to assess single-particle reconstructions of macromolecular complexes ${ }^{15-17}$. We have used the FRC resolution to analyze the trade-off between localization uncertainty and labeling density,

\footnotetext{
${ }^{1}$ Quantitative Imaging Group, Delft University of Technology, Delft, The Netherlands. ${ }^{2}$ Department of Physics and Astronomy, University of New Mexico, Albuquerque, New Mexico, USA. ${ }^{3}$ Department of NanoBiophotonics, Max Planck Institute for Biophysical Chemistry, Göttingen, Germany. ${ }^{4}$ Division of Cell Biology, The Netherlands Cancer Institute, Amsterdam, The Netherlands. ${ }^{5}$ Department of Biochemistry and Molecular Pharmacology, University of Massachusetts Medical School, Worcester, Massachusetts, USA. ${ }^{6}$ RNA Therapeutics Institute, University of Massachusetts Medical School, Worcester, Massachusetts, USA. ${ }^{7}$ These authors contributed equally to this work. Correspondence should be addressed to B.R. (b.rieger@tudelft.nl) or S.S. (s.stallinga@tudelft.nl).

RECEIVED 27 DECEMBER 2012; ACCEPTED 21 MARCH 2013; PUBLISHED ONLINE 28 APRIL 2013; D0I:10.1038/NMETH.2448
} 
Figure 1 | The FRC principle and trade-off between localization uncertainty and labeling density. (a) All localizations are divided into two halves, and the correlation between their Fourier transforms over the perimeter of the circle in Fourier space of radius $q$ is calculated for each $q$, resulting in an FRC curve indicating the decay of the correlation with spatial frequency. The image resolution is the inverse of the spatial frequency for which the FRC curve drops below the threshold $1 / 7 \approx 0.143$, so a threshold value at $q=0.04 \mathrm{~nm}^{-1}$ is equivalent to a $25-\mathrm{nm}$ resolution. Error bars indicate theoretically expected s.d. (Supplementary Note 1). (b) Simulated localization microscopy image of a line pair with mean labeling density $\rho=2.5 \times 10^{3}$ per $\mu \mathrm{m}^{2}$ in the area occupied by the lines and localization uncertainty $\sigma=7.6 \mathrm{~nm}$ (line distance $70 \mathrm{~nm}$, cosinesquared cross-section). (c) Constant resolution in theory (lines) and simulation data (circles) for line pairs as in $\mathbf{b}$ as a function of localization uncertainty and labeling density. Regions of localization uncertaintylimited resolution (blue) and labeling density-limited resolution (yellow) are separated by the red line $\rho \sigma^{2}=e /(6 \pi)$. (d) Simulation results for localization uncertainty versus image resolution for different fixed total measurement times. Camera frame rates were varied to match the on-times of the emitter. The minima of the curves fall on the line $R=2 \pi \sigma$ that separates the yellow region (not enough emitters localized) from the blue region (emitters not localized precisely enough).

and we have applied it to monitor resolution buildup during data acquisition and to compare different localization algorithms. Quantification of the spatial correlations in the image leading to this resolution measure also provides a means to estimate the average number of localizations per emitter contributing to the image. Software for computing the FRC curve and the image resolution for localization microscopy data is available in the form of an ImageJ plugin and Matlab code at http://www.diplib. org/add-ons/ and as Supplementary Software.

\section{RESULTS}

To compute the FRC resolution, we divide the set of single-emitter localizations that constitute a super-resolution image into two statistically independent subsets, which yields two subimages $f_{1}(\vec{r})$ and $f_{2}(\vec{r})$, where $\vec{r}$ denotes the spatial coordinates. Subsequent statistical correlation of their Fourier transforms $\widehat{f_{1}}(\vec{q})$ and $\widehat{f_{2}}(\vec{q})$ over the pixels on the perimeter of circles of constant spatial frequency with magnitude $q=|\vec{q}|$ gives the $\mathrm{FRC}^{16}$

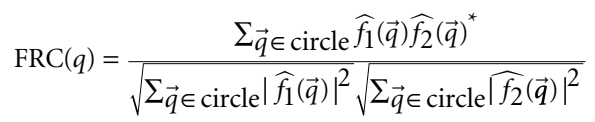

For low spatial frequencies, the FRC curve is close to unity; and for high spatial frequencies, noise dominates the data and the FRC decays to 0 . The image resolution is defined as the inverse of the spatial frequency for which the FRC curve drops below a given threshold. We evaluated different threshold criteria used in the field of cryo-EM ${ }^{15,18-20}$ and found that the fixed threshold equal to $1 / 7 \approx 0.143$ (ref. 18 ) is most appropriate for localization microscopy images (Supplementary Fig. 1). The FRC resolution concept and the steps needed to compute it are illustrated in Figure 1a. FRC resolution describes the length scale below which the image lacks signal content; smaller details are not resolved in the image. Resolution values will always be larger than those based on localization uncertainty or labeling density alone (Supplementary Fig. 2).
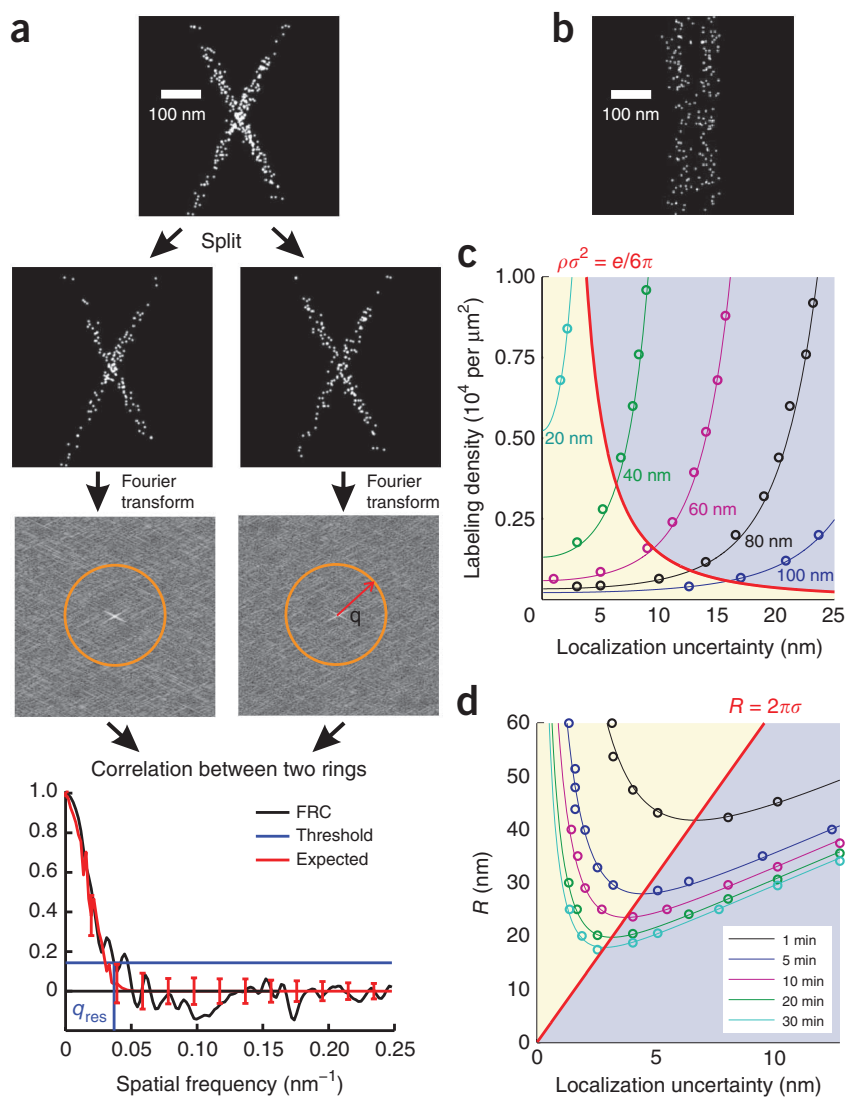

\section{Theoretical considerations and simulations}

FRC resolution allows predictions to be made about the impact of different imaging and sample parameters on the achievable resolution; these predictions are based on the expectation value of the FRC curve (Supplementary Figs. 3 and 4 and Supplementary Note 1), which is given by

$$
\langle\operatorname{FRC}(q)\rangle=\frac{\sum_{\vec{q} \in \operatorname{circle}}\left(Q+N|\widehat{\psi}(\vec{q})|^{2}\right) \exp \left(-4 \pi^{2} \sigma^{2} q^{2}\right)}{\sum_{\vec{q} \in \operatorname{circle}^{[}\left[2+\left(Q+N|\widehat{\psi}(\vec{q})|^{2}\right) \exp \left(-4 \pi^{2} \sigma^{2} q^{2}\right)\right]}}
$$

where $N$ is the total number of localized emitters, $\sigma$ is the average localization uncertainty and $\vec{\psi}(\vec{q})$ denotes the Fourier spectrum of the spatial distribution of the fluorescent emitters. The parameter $Q$ is a measure for spurious correlations due to, for example, repeated photoactivation of the same emitter. Each emitter contributing to the image is localized once for $Q=0$ and in general $Q /\left(1-e^{-Q}\right)$ times on average, provided the emitter activation follows Poisson statistics. Careful analysis of the spatiotemporal correlations in the image and the emitter activation statistics (including effects of photobleaching) can provide a way to estimate $Q$ and correct for its effect on image resolution as well as to estimate the number of fluorescent labels contributing to the image, as is discussed below.

Analytical expressions for the resolution can be derived for particular object types (such as line pairs) often used in resolution definitions (Supplementary Note 2). The resolution $R$ for an image consisting of two parallel lines with a cosine-squared 
Figure 2 | The effect of localization density and data processing on resolution. (a) Localization microscopy image of tubulin labeled with Alexa Fluor 647 in a HeLa cell $(R=58 \pm 1 \mathrm{~nm}$ for the whole image, where uncertainty reflects s.e.m. of 20 random repeats of FRC resolution calculation). Acquisition time was $T=12$ min within $1.4 \times 10^{4}$ frames, the localization uncertainty was $\sigma=9.7 \mathrm{~nm}$ after merging nearby localizations in subsequent frames (Online Methods) and the density of localizations was $\rho=6.0 \times 10^{2}$ per $\mu \mathrm{m}^{2}$. (b-e) Magnified insets of two crossing filaments (upper boxed region in a) constructed from fewer time frames showing poorer resolution (indicated by the distance between the blue arrows). (f) Resolution $(R)$ buildup during acquisition, with $R=2 \pi \sigma$ plotted in blue, showing a transition from density-limited to precision-limited resolution. (g-i) Reconstructions of lower boxed region in a by different localization algorithms showing maximumlikelihood estimation (g; MLE, $R=58 \pm 1 \mathrm{~nm}$ ), least squares fitting (h; $L S, R=60 \pm 1 \mathrm{~nm}$ ) and centroid fitting (i; CEN, $R=88 \pm 2 \mathrm{~nm}$ ). (j) Localization microscopy image of the actin cytoskeleton (F-actin) of a fixed HeLa cell labeled with phalloidin coupled to Alexa Fluor 647 after correction for sample drift of $~ 70-100 \mathrm{~nm}$ during acquisition. The image was obtained from $5.0 \times 10^{4}$ frames in $8 \mathrm{~min}(\sigma=8.0 \mathrm{~nm}$, $\left.\rho=8.2 \times 10^{3} \mu \mathrm{m}^{-2}, \rho \sigma^{2}=0.52,2 \pi \sigma=50 \mathrm{~nm}\right)$. $(\mathbf{k}-\mathbf{n})$ Magnified insets of reconstructions before $(\mathbf{k}, \mathbf{l}$; left boxed region in $\mathbf{j})$ and after drift correction $(\mathbf{m}, \mathbf{n}$; right boxed region in $\mathbf{j})$. Resolutions before and after drift correction were $R=79 \pm 2 \mathrm{~nm}$ and $R=54 \pm 1 \mathrm{~nm}$, respectively. The arrows indicate regions of sharper detail after drift correction.
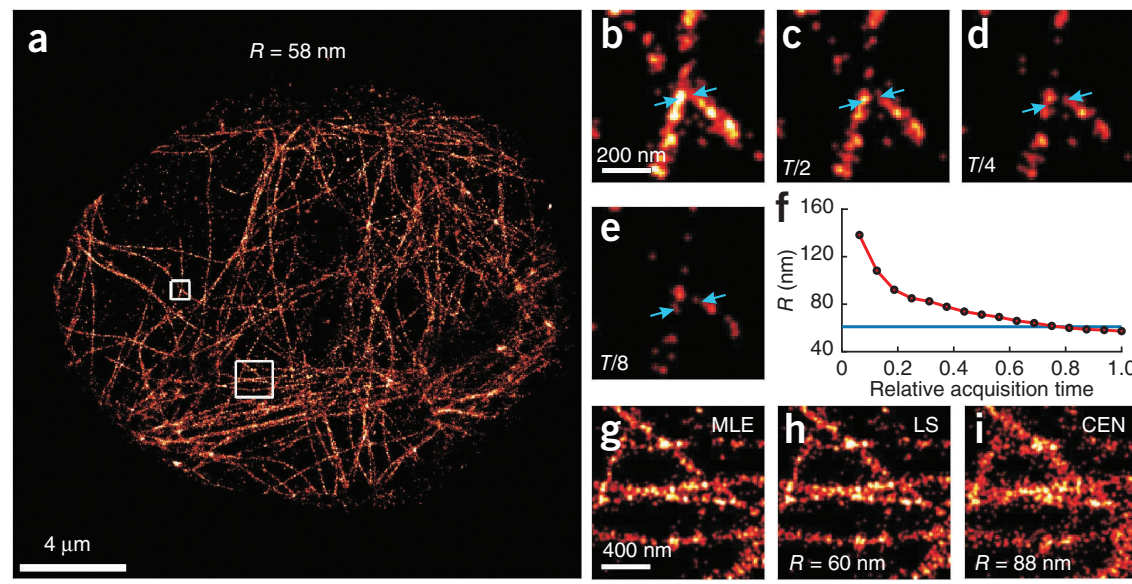

f 160 .
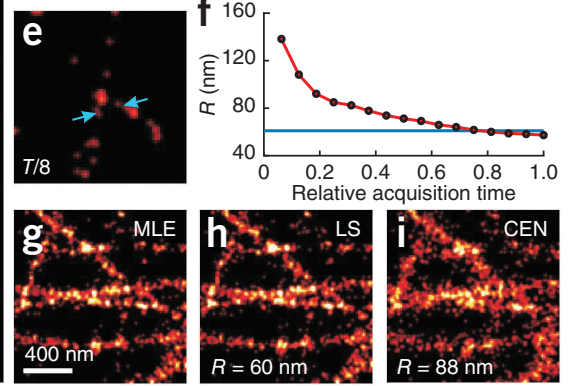

Without drift correction

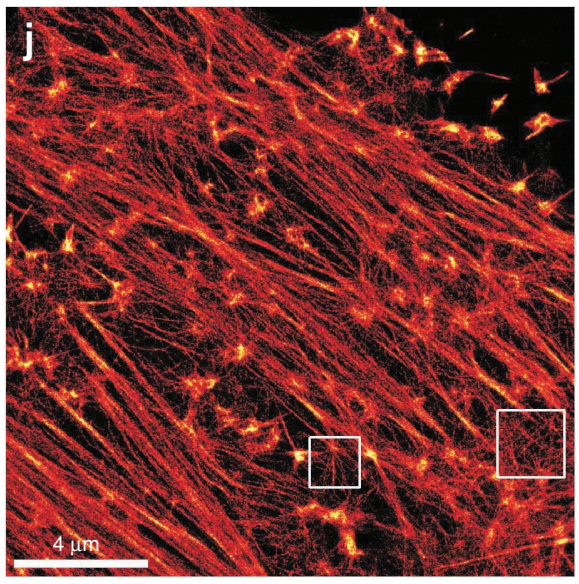

With drift correction

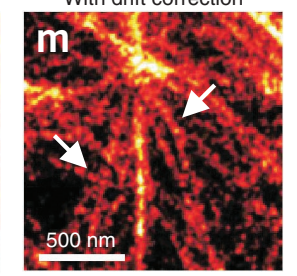

$R=54 \mathrm{~nm}$
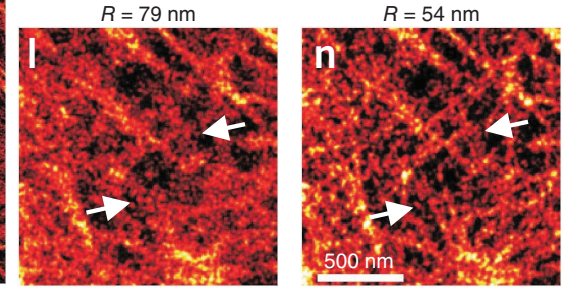

cross-section and mean labeling density $\rho$ in the area occupied by the lines is

$$
R=\frac{2 \pi \sigma}{\sqrt{W\left(6 \pi \rho \sigma^{2}\right)}}
$$

where $W(x)$ is the Lambert $W$-function ${ }^{21}$. Two regimes can be identified in which changes in either labeling density or localization uncertainty have the most impact on improving the resolution. At the boundary between these regimes, the relative gains in resolution due to changes in either quantity are equally large. This trade-off occurs at $R=2 \pi \sigma$ (Supplementary Note 3 ), which corresponds to

$$
\rho \sigma^{2}=\frac{e}{6 \pi} \approx 0.14
$$

The region $\rho \sigma^{2}<e /(6 \pi)$ is labeling-density limited, whereas $\rho \sigma^{2}>e /(6 \pi)$ is localization-uncertainty limited (Fig. 1b,c). The exact boundary between the two regimes depends on the underlying object, so the boundary value for the two-line example serves only as a rule of thumb (Supplementary Note 3). For example, for $M$ parallel lines, we obtain a value $e /(3 \pi M)$. From this it may be inferred that the trade-off occurs for a value smaller than 0.14 for any intricate but irregular object structure.

The same trade-off as above may also manifest itself in the optimization of image resolution, given a fixed total acquisition time (Fig. 1b,d). Suppose that the photon count per localization is improved by increasing the on-times of the emitters while keeping the emitters' brightness and the number of simultaneously active emitters constant: this then also reduces the total number of labels that can be localized in a given acquisition time. Therefore, longer single-emitter events yield more accurate localizations, but at the expense of a lower recorded emitter density ${ }^{3,22}$. Again, the optimum is $R=2 \pi \sigma$, independent of the object (Supplementary Note 3). Tuning the on-times as described here may be done in the design phase of an experiment by the choice of label or buffer composition.

\section{Resolution buildup during data acquisition}

To test and evaluate the FRC resolution measure, we imaged tubulin networks in fixed HeLa cells labeled with Alexa Fluor 647 using localization microscopy (Fig. 2a and Online Methods).

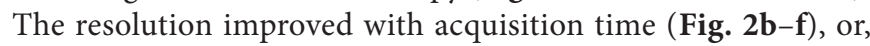
equivalently, with the density of localized labels. The trade-off point between the localization density and uncertainty limited regimes lay at $R=2 \pi \sigma=61 \mathrm{~nm}$. Therefore, the resolution values for Figures $\mathbf{2 b}$-e were labeling-density limited, and the trade-off point was just crossed at the end of the data acquisition. Real-time monitoring of the resolution buildup by real-time single-molecule fitting algorithms $\mathrm{s}^{23}$ provides a much needed stopping criterion for localization microscopy data acquisitions.

The FRC resolution concept is also sensitive to differences in localization uncertainty (Fig. 2g-i). Maximum-likelihood estimation $(R=58 \pm 1 \mathrm{~nm})$ is theoretically optimal ${ }^{24}$ and is slightly better than least-squares fitting $(R=60 \pm 1 \mathrm{~nm})$ and superior to centroid fitting $(R=88 \pm 2 \mathrm{~nm})$. All specified uncertainties are computed from 20 FRC resolution estimates obtained from different random assignments of localizations to half data sets (s.e.m.). Because the 
Figure 3 | Spurious correlations from a two-color localization microscopy image. (a) Overview image of a tubulin network labeled with both Alexa Fluor 647 (magenta) and Alexa Fluor 750 (green). The inset shows the quality of registration. (b) Uncorrected FRC curves for the magenta and green channels are higher than that for the cross-channel because of spurious correlations from repeated photoactivations of individual emitters, which result in overly optimistic resolution values $(R=25 \pm 1 \mathrm{~nm}$ and $34 \pm 1 \mathrm{~nm}$, respectively, compared to $118 \pm 2 \mathrm{~nm}$ for the cross-channel). Uncertainty reflects s.e.m. of 20 random repeats of the FRC resolution calculation. (c) FRC curves corrected for spurious correlations all yield similar resolution values $(108 \pm 1 \mathrm{~nm}$ for Alexa Fluor 647, $133 \pm 2 \mathrm{~nm}$ for Alexa Fluor 750, $121 \pm 2 \mathrm{~nm}$ for the crosschannel). (d-f) Scaled FRC numerator curves showing a plateau for intermediate spatial frequencies, which is used to estimate the correction term and $Q$ parameter. For this correction (Online Methods) we used a mean and width of the distribution of localization uncertainties equal to $9.2 \mathrm{~nm}$ and $2.8 \mathrm{~nm}$ for Alexa Fluor 647 and $12 \mathrm{~nm}$ and $2.0 \mathrm{~nm}$ for Alexa Fluor 750.

effect of the parameter $Q$ on the resolution for this data set was found to be negligible, it was not necessary to correct for it.

Sample drift is a common annoyance in optical nanoscopy, as motion has to be limited to a few nanometers over typical acquisition times of many minutes. We analyzed the drift in localization microscopy data of the actin cytoskeleton of a fixed HeLa cell labeled with phalloidin coupled to Alexa Fluor 647 (Fig. 2j) without the use of fiducial mark$\mathrm{ers}^{25}$. A drift of $\sim 70-100 \mathrm{~nm}$ was found with this procedure and corrected for. Computed resolution values before drift correction (Fig. 2k,l; $R=79 \pm 1 \mathrm{~nm}$ ) were much worse than those after drift correction (Fig. $2 \mathbf{m}, \mathbf{n} ; R=54 \pm 1 \mathrm{~nm}$ ), which is in agreement with the apparent detail in the images (Fig. 2k-n). For this data set also, the effect of $Q$ was found to be negligible. More experimental data are provided to show the validity of the FRC resolution (Supplementary Figs. 5-7).

\section{Estimation of the number of localizations per emitter}

Multiple localizations per emitter due to, for example, repeated photoactivations lead to spurious correlations between the two image halves, resulting in overoptimistic resolution values. This is particularly problematic for cases involving large numbers of localizations per emitter, low localization uncertainties and low labeling densities (Supplementary Figs. 8 and $\mathbf{9}$ ).

The FRC can be corrected for this effect by estimating the spurious correlation parameter $Q$ in equation (2). To that end, we divided the numerator of the FRC by the weighted average of the function $\exp \left(-4 \pi^{2} \sigma^{2} q^{2}\right)$ over the distribution of localization uncertainties. The parameter $Q$ is proportional to the minimum of that curve, which takes the form of a broad plateau if $Q>>1$ (Online Methods). To test this method, we analyzed a two-color image of tubulin labeled with both Alexa Fluor 647 and Alexa Fluor 750 (ref. 26; Fig. 3a-c). The resolution values for Alexa
Fluor 647 and Alexa Fluor 750 without correction $(25 \pm 1 \mathrm{~nm}$ and $34 \pm 1 \mathrm{~nm}$, respectively) were much lower than the resolution derived from the cross-channel, that is, when taking the two color images as data halves for the FRC $(118 \pm 2 \mathrm{~nm})$. This difference was due to the multiple localizations per emitter, which affect the one-color FRC curves but not the cross-channel curve. The FRC curves and attendant resolution values were much more similar after correction. The remaining differences in the calculated resolution values reflected the differences in labeling density (the density of localizations was $4.0 \times 10^{3}$ per $\mu \mathrm{m}^{2}$ for Alexa Fluor 647 and $1.3 \times 10^{3}$ per $\mu \mathrm{m}^{2}$ for Alexa Fluor 750$)$ and localization uncertainty ( $9.2 \mathrm{~nm}$ and $12 \mathrm{~nm}$, respectively).

We checked the data sets of Figure 2a,j for spurious correlations and found $Q=0.28$ and $Q=0.33$, respectively, which led to corrected resolution values equal to $62 \pm 2 \mathrm{~nm}$ and $66 \pm 1 \mathrm{~nm}$, respectively. This means that neglecting to correct for spurious correlations gave rise to an underestimation of the resolution value by only several nanometers. These estimated values for $Q$ are smaller than the values for the data set of Figure 3 primarily because $Q$ scales with the data acquisition time, which is much smaller in Figure 2a,j than the $1.4 \times 10^{5}$ frames in $39 \mathrm{~min}$ and $3.0 \times 10^{4}$ frames in $25 \mathrm{~min}$ for Alexa Fluor 647 and Alexa Fluor 750, respectively (Fig. 3). Other reasons for the discrepancy of $Q$ values may be found in differences in photobleaching behavior and preprocessing for candidate selection of single-emitter events (from false positives, for example). Finally, the density of localizations for Figure $2 \mathbf{j}$ is close to $10^{4}$ per $\mu \mathrm{m}^{2}, 1-2$ orders of magnitude larger than the density in other data sets. In the limit of high labeling density, the effects of spurious correlations are negligible compared to the intrinsic image correlations 
Figure $4 \mid 3 D$ resolution. (a) Representation of a 3D localization microscopy image of a tubulin network, with the axial coordinate in false color. (b) Orthogonal slices of the Fourier plane correlation (FPC). (c-e) Crosssections of the FPC for this data set in the $q_{x} q_{z}$ plane (c), $q_{y} q_{z}$ plane (d) and $q_{x} q_{y}$ plane (e), with added threshold contours for $\mathrm{FPC}=1 / 7$ (black lines). The FPC clearly shows the anisotropy of image content resulting from the line-like structure of the filaments (the highest image resolution is perpendicular to the filaments) as well as from the anisotropy in localization uncertainty (the lowest resolution is in the axial direction).
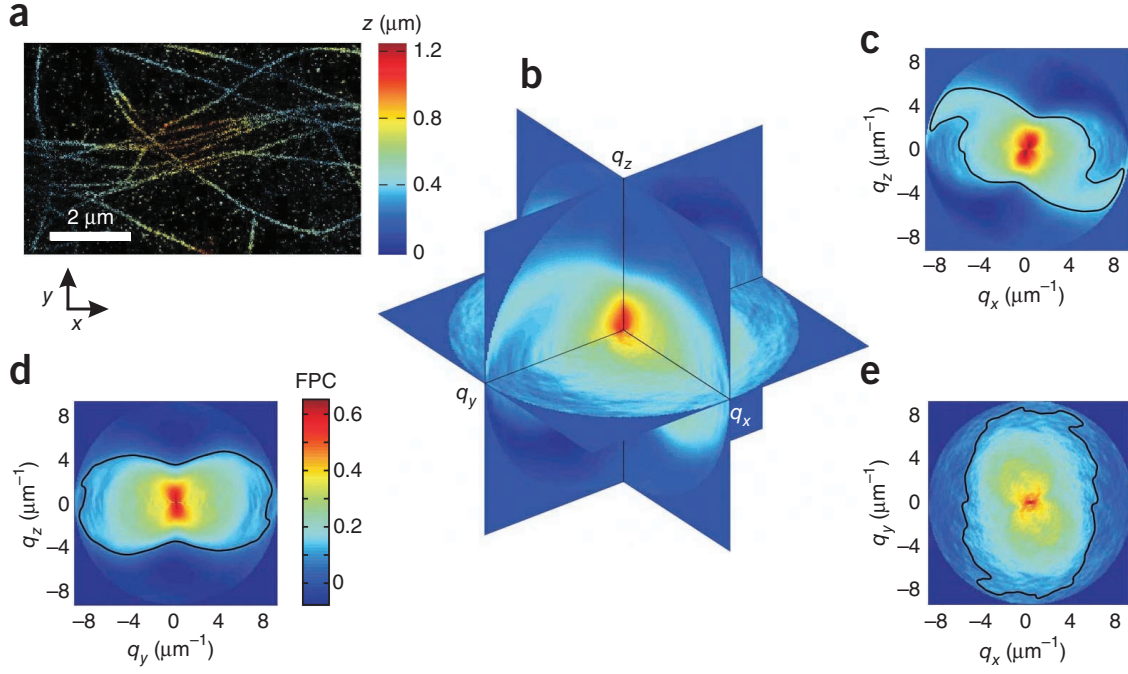

$q_{x}\left(\mu \mathrm{m}^{-1}\right)$

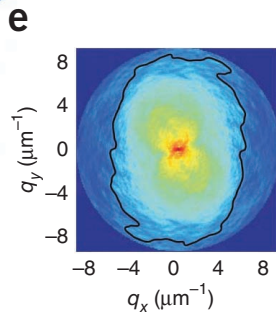

(Supplementary Notes $\mathbf{1}$ and 2 ). We point out that the correction method appears to be quite sensitive to (the distribution of) the localization uncertainty, and to any residual effects of drift, and must therefore be applied with care.

image of a tubulin network labeled with Alexa Fluor 647 (Fig. 4) using the bifocal method ${ }^{33}$ shows clear anisotropy with filaments oriented mostly in the $x y$ plane along the $x$ direction. Therefore, the FPC is highest in the $y$ direction, orthogonal to the filaments, and worst in the $z$ direction.

The FLC for part of the data set of Figure $2 \mathbf{j}$ shows that the region of resolved spatial frequencies is anisotropic and highest in the direction orthogonal to the filaments (Supplementary Fig. 11), as expected. Another way in which the FRC resolution concept can be generalized targets local variations in the density of the sample's spatial structure. Local image resolution can be obtained from resolution values of overlapping subimage patches (Supplementary Fig. 12).

\section{DISCUSSION}

The FRC resolution concept can be naturally extended to STED, imaging with an extended diffraction limit such as structured illumination microscopy ${ }^{34}$, and conventional confocal and wide-field imaging. It is possible not only to conceptually extend the FRC method but also to measure the resolution directly from experimental data. This stands in contrast to recently introduced unified resolution concepts $\mathrm{s}^{35}$, which provide only a rigorous theoretical framework. The FRC resolution is most easily computed from two images of the same scene that differ only in noise content. The resolution then depends on the signal-to-noise ratio, spectral image content and (effective) optical transfer function. The width of the effective point-spread function replaces the role of the localization uncertainty. In the limit of infinitely high signal-tonoise ratio, the FRC resolution reduces to Abbe's diffraction limit (for the conventional fluorescence imaging modalities) or to the limit that has been proposed for STED ${ }^{36}$ (Supplementary Note 4 and Supplementary Fig. 13). We also applied the FRC concept to time-lapse recordings in scanning electron microscopy in single-electron counting mode (Supplementary Fig. 14). For any extension of the FRC concept, systematic dependencies between image halves due to, for example, fixed-pattern noise or common alignment references must be prevented. Alignment references have caused particular problems for the application of the FRC concept in the field of single-particle cryo-EM ${ }^{37}$.

We envision that FRC resolution may be used for characterizing and optimizing fluorescence labeling and data processing 
strategies in general. The FRC resolution may be used to rate different approaches for faster super-resolution image buildup that deal with high densities of simultaneously active emitters ${ }^{38-40}$. Access to the number of molecules in a multimolecular complex, such as the spliceosome or transcription machinery, without the need to make assumptions about their spatial structure adds a new dimension to the application of optical nanoscopy with reversibly switchable fluorescent dyes. Most notably, a resolution measure as proposed here is indispensable for advancing the blooming field of optical nanoscopy because it provides a quantitative guide for reliable interpretation of data, thus enabling sound biological conclusions.

\section{METHODS}

Methods and any associated references are available in the online version of the paper.

Note: Supplementary information is available in the online version of the paper.

\section{ACKNOWLEDGMENTS}

We thank K. Jalink for encouragement and support; S. Schwartz, F. Huang, J. Byars and S. Liu for assistance with experiments; and V. van Ravesteijn and P. Kruit for providing scanning electron microscope data. We appreciate the thoughtful comments of T. Young and L. van Vliet. R.P.J.N. and D.L.P. are supported by the Dutch Technology Foundation STW, which is part of the Netherlands Organisation for Scientific Research (NWO) and which is partly funded by the Ministry of Economic Affairs, Agriculture and Innovation. K.A.L. was supported by US National Science Foundation CAREER Award \#0954836 and US National Institutes of Health grant P50GM085273.

\section{AUTHOR CONTRIBUTIONS}

R.P.J.N., S.S. and B.R. devised the conceptual framework and derived theoretical results. Simulations were done by R.P.J.N. Experimental data sets were acquired by R.P.J.N. (Fig. 2a-i), D.L.P. (Fig. 2j-n), K.A.L. (Figs. $2 \mathrm{a}-\mathbf{i}$ and $\mathbf{4}$ ) and M.B. (Fig. 3). Data were analyzed by R.P.J.N., M.B., S.S. and B.R. D.G. provided research advice. The paper was written by R.P.J.N., D.G., S.S. and B.R.

\section{COMPETING FINANCIAL INTERESTS}

The authors declare no competing financial interests.

Reprints and permissions information is available online at http://www.nature. com/reprints/index.html.

1. Hell, S.W. \& Wichmann, J. Breaking the diffraction resolution limit by stimulated emission: stimulated-emission-depletion microscopy. Opt. Lett. 19, 780-782 (1994).

2. Hofmann, M., Eggeling, C., Jakobs, S. \& Hell, S.W. Breaking the diffraction barrier in fluorescence microscopy at low light intensities by using reversibly photoswitchable proteins. Proc. Natl. Acad. Sci. USA 102, 17565-17569 (2005).

3. Betzig, E. et al. Imaging intracellular fluorescent proteins at nanometer resolution. Science 313, 1642-1645 (2006).

4. Rust, M.J., Bates, M. \& Zhuang, X. Sub-diffraction-limit imaging by stochastic optical reconstruction microscopy (STORM). Nat. Methods 3, 793-795 (2006).

5. Fölling, J. et al. Fluorescence nanoscopy by ground-state depletion and single-molecule return. Nat. Methods 5, 943-945 (2008).

6. Heilemann, M. et al. Subdiffraction-resolution fluorescence imaging with conventional fluorescent probes. Angew. Chem. Int. Ed. Engl. 47, 6172-6176 (2008).

7. Lidke, K., Rieger, B., Jovin, T.M. \& Heintzmann, R. Superresolution by localization of quantum dots using blinking statistics. Opt. Express 13, 7052-7062 (2005).

8. Dertinger, T., Colyer, R., Iyer, G., Weiss, S. \& Enderlein, J. Fast, background-free, 3D super-resolution optical fluctuation imaging (SOFI). Proc. Natl. Acad. Sci. USA 106, 22287-22292 (2009).

9. Ram, S., Ward, E.S. \& Ober, R.J. Beyond Rayleigh's criterion: a resolution measure with application to single-molecule microscopy. Proc. Natl. Acad. SCi. USA 103, 4457-4462 (2006).

10. Löschberger, A. et al. Super-resolution imaging visualizes the eightfold symmetry of gp210 proteins around the nuclear pore complex and resolves the central channel with nanometer resolution. J. Cell Sci. 125, 571-575 (2012).
11. Kanchanawong, P. et al. Nanoscale architecture of integrin-based cell adhesions. Nature 468, 580-584 (2010).

12. van de Linde, S., Wolter, S., Heilemann, M. \& Sauer, M. The effect of photoswitching kinetics and labeling densities on super-resolution fluorescence imaging. J. Biotechnol. 149, 260-266 (2010).

13. Cordes, T. et al. Resolving single-molecule assembled patterns with superresolution blink-microscopy. Nano Lett. 10, 645-651 (2010).

14. Fitzgerald, J.E., Lu, J. \& Schnitzer, M.J. Estimation theoretic measure of resolution for stochastic localization microscopy. Phys. Rev. Lett. 109, 048102 (2012).

15. Saxton, W.0. \& Baumeister, W. The correlation averaging of a regularly arranged bacterial cell envelope protein. J. Microsc. 127, 127-138 (1982).

16. Van Heel, M. Similarity measures between images. Ultramicroscopy 21, 95-100 (1987).

17. Unser, M., Trus, B.L. \& Steven, A.C. A new resolution criterion based on spectral signal-to-noise ratios. Ultramicroscopy 23, 39-51 (1987).

18. Beckmann, R. et al. Alignment of conduits for the nascent polypeptide chain in the ribosome-Sec61 complex. Science 278, 2123-2126 (1997).

19. Böttcher, B., Wynne, S.A. \& Crowther, R.A. Determination of the fold of the core protein of hepatitis B virus by electron cryomicroscopy. Nature 386, 88-91 (1997).

20. Rosenthal, P.B. \& Henderson, R. Optimal determination of particle orientation, absolute hand, and contrast loss in single-particle electron cryomicroscopy. J. Mol. Biol. 333, 721-745 (2003).

21. Barry, D.A. et al. Analytical approximations for real values of the Lambert W-function. Math. Comput. Simul. 53, 95-103 (2000).

22. Small, A.R. Theoretical limits on errors and acquisition rates in localizing switchable fluorophores. Biophys. J. 96, L16-L18 (2009).

23. Wolter, S. et al. rapidSTORM: accurate, fast open-source software for localization microscopy. Nat. Methods 9, 1040-1041 (2012).

24. Smith, C.S., Joseph, N., Rieger, B. \& Lidke, K.A. Fast, single-molecule localization that achieves theoretically minimum uncertainty. Nat. Methods 7, 373-375 (2010).

25. Mlodzianoski, M.J. et al. Sample drift correction in 3D fluorescence photoactivation localization microscopy. Opt. Express 19, 15009-15019 (2011).

26. Bates, M., Dempsey, G.T., Chen, K.H. \& Zhuang, X. Multicolor superresolution fluorescence imaging via multi-parameter fluorophore detection. ChemPhysChem 13, 99-107 (2012).

27. Lando, D. et al. Quantitative single-molecule microscopy reveals that CENP-A(Cnp1) deposition occurs during G2 in fission yeast. Open Biol. 2, 120078 (2012).

28. Sengupta, P. et al. Probing protein heterogeneity in the plasma membrane using PALM and pair correlation analysis. Nat. Methods 8, 969-975 (2011).

29. Veatch, S.L. et al. Correlation functions quantify super-resolution images and estimate apparent clustering due to over-counting. PLOS ONE 7, e31457 (2012).

30. Annibale, P., Vanni, S., Scarselli, M., Rothlisberger, U. \& Radenovic, A. Identification of clustering artifacts in photoactivated localization microscopy. Nat. Methods 8, 527-528 (2011).

31. Dempsey, G.T., Vaughan, J.C., Chen, K.H., Bates, M. \& Zhuang, X. Evaluation of fluorophores for optimal performance in localization-based super-resolution imaging. Nat. Methods 8, 1027-1036 (2011).

32. von Middendorff, C., Egner, A., Geisler, C., Hell, S.W. \& Schönle, A. Isotropic 3D nanoscopy based on single emitter switching. Opt. Express 16, 20774-20788 (2008).

33. Toprak, E. et al. Defocused orientation and position imaging (DOPI) of myosin V. Proc. Natl. Acad. Sci. USA 103, 6495-6499 (2006).

34. Gustafsson, M.G.L. Surpassing the lateral resolution limit by a factor of two using structured illumination microscopy. J. Microsc. 198, 82-87 (2000).

35. Mukamel, E.A. \& Schnitzer, M.J. Unifed resolution bounds for conventional and stochastic localization fluorescence microscopy. Phys. Rev. Lett. 109, 168102 (2012).

36. Hell, S.W. Towards fluorescence nanoscopy. Nat. Biotechnol. 21, 1347-1355 (2003).

37. Scheres, S.H. \& Chen, S. Prevention of overfitting in cryo-EM structure determination. Nat. Methods 9, 853-854 (2012).

38. Huang, F., Schwartz, S.L., Byars, J.M. \& Lidke, K.A. Simultaneous multipleemitter fitting for single molecule super-resolution imaging. Biomed. Opt. Express 2, 1377-1393 (2011).

39. Holden, S.J., Uphoff, S. \& Kapanidis, A.N. DAOSTORM: an algorithm for high-density super-resolution microscopy. Nat. Methods 8, 279-280 (2011).

40. Zhu, L., Zhang, W., Elnatan, D. \& Huang, B. Faster STORM using compressed sensing. Nat. Methods 9, 721-723 (2012). 


\section{ONLINE METHODS}

Computation of FRC and FRC resolution. Computation. The starting point for the computation of the FRC resolution is a set of estimated fluorophore locations along with the numbers of the frames from which they originate. To calculate the resolution from a set of localizations $\left\{\vec{r}_{i}\right\}$, the following steps were followed for experimental data:

1. The set of $N$ localizations was divided into two half sets $N_{1}$ and $N_{2}$ of size $N / 2$ by splitting the time series into blocks of 500 frames and assigning an equal number of blocks randomly to each half set. Alternatively, half sets could also have been obtained by simply assigning localizations randomly to half sets or by splitting the time series in two parts.

2. The localizations from each half set were binned into images $f_{1}$ and $f_{2}$. For the experimental data, the bin sizes (that is the superresolution pixel sizes) were taken to be $p$ times smaller than the camera pixel size, typically $p=10(10-\mathrm{nm}$ back-projected pixel size). See Supplementary Note 2 for a further discussion about the choice of pixel size.

3. An intensity mask $M(\vec{r})$ was applied to the binned images to taper the edges. For this work, a Tukey window ${ }^{41}$ was used, which has the form $M(\vec{r})=m(x) m(y)$, where

$$
m(x)=\left\{\begin{array}{cl}
\sin ^{2}(4 \pi x / L) & \text { if } x<\frac{L}{8} \text { and } x>7 L / 8 \\
1 & \text { if } x \geq \frac{L}{8} \text { and } x \leq 7 L / 8
\end{array}\right.
$$

Here $L$ denotes the size of the field of view.

4. Both binned images were Fourier transformed.

5. The FRC was obtained for spatial frequencies $q=1 / L, 2 / L, \ldots$ by evaluating equation (1), where $\vec{q} \in$ circle means the set $\{\vec{q} \mid q \leq$ $|\vec{q}|<q+\delta q\}$ where $\delta q=1 / L$ is the pixel size in Fourier space.

6. As the FRC curve is often quite noisy, it was smoothed with a LOESS (locally estimated scatter plot smoothing) method $^{42}$ with a second-order polynomial and tri-cube weight function around each $q$ over a span $\Delta q=1 /(20 l)$, where $l$ is the pixel size.

7. The first intersection $q_{\text {res }}$ between the resulting smoothed FRC curve and the threshold was used to finally calculate the resolution $R=1 / q_{\text {res }}$.

Please note that the term $\sum \vec{q} \in$ circle $\widehat{f_{1}}(\vec{q}) \widehat{f_{2}}(\vec{q})^{*}$ is real, if $f_{1}(\vec{r})$ and $f_{2}(\vec{r})$ are real, because then it holds that $\hat{f}(\vec{q})=\hat{f}(-\vec{q})^{*}$ and in each term $f(\vec{q})+f(-\vec{q})$, the complex parts cancel out. Note that we assume square-sized images $f_{1}(\vec{r})$ and $f_{2}(\vec{r})$ for ease of computation. If the images are not square, the images must be extended through zero padding or the circle averaging must be replaced by averaging over ellipses, as the pixel size in Fourier space depends on the linear size of the image. The uncertainty of the FRC resolution value is found by evaluating the resolution for 20 different random splittings of the entire data set. The resulting mean and s.d. are the reported numbers.

Bright spots. Localization microscopy images sometimes contain clusters of localizations with a diameter of a few times the localization precision $\sigma$ where the density of localizations is very high. These clusters may be due to, for example, anomalous fluorescent molecules that are active during a large part of the total measurement time. However, the localizations in these clusters can represent a substantial fraction of all localizations and are very close together. This close spatial proximity translates into large correlations in the high spatial frequency components of the images that are not representative of the other parts of the image. Therefore, these clusters are considered to be artifacts. Thus, it is often necessary to suppress the influence of these clusters. One approach that we adopted to this end was to mask out these clusters if they were not on the main structures. Remaining bright spots were suppressed by the procedure to merge nearby localizations in time, which is further outlined below, and by limiting the number of binned localizations per super-resolution pixel to a maximum of 5 . For the data of Figure 2a, all these approaches were adopted: most bright spots were removed by segmenting the cell in the wide-field image and deleting all localizations outside the cell. For the data in Figure $\mathbf{2} \mathbf{j}$, no masking of regions outside the cell was applied because the entire field of view is filled. For the other experimental data sets, only the merging of nearby localizations was used to reduce the influence of bright spots.

Spurious correlations. Multiple localizations of the same emitter result in substantial correlations at all spatial frequencies. The result is that the numerator of the FRC contains a term $(2 \pi q L) N Q \exp \left(-4 \pi^{2} q^{2} \sigma^{2}\right)$ (or the weighted average of this quantity over the distribution of localization uncertainties) that belongs to the denominator of the FRC. Here $L$ is the size of the field of view. Correction for this effect then requires that this spurious term is estimated and corrected for. The first step in estimating the spurious correlations consisted of calculating the numerator of the FRC and dividing by the number of pixels in the Fourier circle resulting in a function $v(q)$

$$
v(q)=\frac{1}{2 \pi q L} \sum_{\vec{q} \in \operatorname{circle}} \widehat{f_{1}}(\vec{q}) \widehat{f_{2}}(\vec{q})^{*}
$$

Subsequently, $v(q)$ was divided by $H(q) \operatorname{sinc}(\pi q L)^{2}$, where $H(q)$ is the factor in the correlation averages related to the localization uncertainties that depends on the mean $\sigma_{\mathrm{m}}$ and width $\Delta \sigma$ of the distribution of localization uncertainties, which is taken to be Gaussian (Supplementary Note 1)

$$
H(q)=\frac{1}{\sqrt{1+8 \pi^{2} \Delta \sigma^{2} q^{2}}} \exp \left(-\frac{4 \pi^{2} \sigma_{\mathrm{m}}^{2} q^{2}}{1+8 \pi^{2} \Delta \sigma^{2} q^{2}}\right)
$$

Therefore, the result should have an expectation value $(Q+$ $N S(q)) / 4$. Here $S(q)$ is defined formally in Supplementary Note 1 as the ring average of the spectral signal content of the image. For estimation of $N Q / 4$ in a robust manner, the logarithm of $\left|v(q) / H(q) / \operatorname{sinc}(\pi q L)^{2}\right|$ was taken and smoothed, and $\log (N Q / 4)$ was then estimated as the minimum of this smoothed logarithm. The smoothing was accomplished through robust LOESS ${ }^{42}$ with a second-order polynomial and tri-cube weight function around each $q$ over a span $\Delta q=1 /(10 l)$.

The logarithm of $\left|v(q) / H(q) / \operatorname{sinc}(\pi q L)^{2}\right|$ typically is a function that initially decreases, then levels off to a constant plateau value and finally increases again. The mean and width of the assumed Gaussian distribution of localization uncertainties are adjusted to get a horizontal plateau of the largest possible width. This 
procedure, though manually executed, can be used to estimate these parameters with an accuracy of typically $1-2 \mathrm{~nm}$. A plateau results when $Q>N S(q)$, so that $\left|v(q) / H(q) / \operatorname{sinc}(\pi q L)^{2}\right| \approx N Q / 4$. For large $q$, the noise on the absolute value of $v(q)$, which has an expected value of about $N / \sqrt{ }(32 \pi q L)$, is blown up by the factor $1 / H(q) / \operatorname{sinc}(\pi q L)^{2}$. Therefore, the aforementioned procedure will also yield a narrow plateau and a finite estimate for $Q$ even in the case where $Q<<N S(q)$, when there is no plateau due to $Q$. In this case $Q$ will be overestimated and there will therefore be an overcorrection for spurious correlations.

FLC and FPC computation. The Fourier line correlation (FLC, $n=2$ dimensions) or Fourier plane correlation (FPC, $n=3$ dimensions) are evaluated numerically as follows. The entire image is again split into two subimages $f_{1}(\vec{r})$ and $f_{2}(\vec{r})$, with Fourier transforms $\widehat{f_{1}}(\vec{q})$ and $\widehat{f_{2}}(\vec{q})$. The FLC and FPC are defined similarly to the FRC

$$
\frac{G_{12}(\vec{q})}{\sqrt{G_{11}(\vec{q}) G_{22}(\vec{q})}}
$$

where the correlation averages are now defined as averages over lines $(n=2)$ or planes $(n=3)$ perpendicular to $\vec{q}$

$$
G_{j l}(\vec{q})=\sum_{\vec{q}^{\prime} \in \text { line, plane }} \widehat{f_{j}}\left(\vec{q}^{\prime}\right) \widehat{f}_{l}\left(\vec{q}^{\prime}\right)^{*}, j, l=\{1,2\}
$$

where the summation over the line/plane means $\vec{q}^{\prime} \in\left\{\vec{q}^{\prime} \mid\left(\vec{q}^{\prime} \pm \vec{q}\right)\right.$. $\vec{q}=0\}$ (Supplementary Fig. 15). For $n=2$ the implementation of a line average boils down to a Radon transform, executed with the Matlab (MathWorks) function 'radon'. For $n=3$ the plane average is done by first rotating $\widehat{f_{1}}(\vec{q})$ and $\widehat{f_{2}}(\vec{q})$ to a grid with $\vec{q}$ oriented along the $z$ axis, executed with the function 'rotation3D' of the DipImage toolbox (http://www.diplib.org/), and subsequent averaging over the $x$ and $y$ directions in the rotated frame. Averaging over lines/planes with an orientation that is not aligned with one of the coordinate axes is possible but computationally much more costly than the rotation procedure. Computational time can be saved by doing the 3D Fourier transforms to get $\widehat{f_{1}}(\vec{q})$ and $\widehat{f_{2}}(\vec{q})$ on the full data cube of $L \times L \times L$ super-resolution pixels and all the rotations on a cropped $M \times M \times M$ cube, where $M$ is adjusted so that the FPC drops below the threshold close to the edge of the cropped data cube. For the data set of Figure 4, we used $L=1,024, M=191$ and 10-nm super-resolution pixels. Note that square/cubic image sizes are used for convenience. Only square/cubic images have isotropic pixel sizes in Fourier space if the pixel sizes in real space are isotropic. Further information on the computation of FRC and FRC resolution is provided in Supplementary Note 5.

Simulations. General setup. Simulations were conducted in Matlab with the use of the image processing toolbox DipImage and several C-language and CUDA codes that were compiled to Matlab MEX files and run from within the Matlab environment. Localization microscopy data without multiple localizations per emitter $(Q<<1)$ are simulated as follows. Poisson noise is added to an object image serving as the 'ground truth' (which has pixel values larger than 0 ). The resulting value per pixel is taken to be the simulated number of emitters in that pixel, and a number of random points equal to this value is generated for each pixel.
Each of these points is then displaced according to a zero-mean normal distribution with variance $\operatorname{Var}(\Delta x)=\operatorname{Var}(\Delta y)=\sigma^{2}$ to obtain the simulated data (localizations). Localization microscopy data with multiple localizations per emitter are simulated in a similar way. The only difference is that each of the simulated emitter positions is used to generate a binomially distributed number $M_{j}$ of offspring points (localizations) instead of 1. All of these offspring points are then displaced with a zero-mean normal distribution with variance $\operatorname{Var}(\Delta x)=\operatorname{Var}(\Delta y)=\sigma^{2}$.

Figure $\mathbf{1 b}$. The result in Figure $\mathbf{1 b}$ was obtained by simulating measurements without multiple localizations per emitter $(Q<<1)$ for ground truth images of the form (Supplementary Fig. 16)

$$
o(x, y)=\left\{\begin{array}{cc}
\rho(1-\cos (2 \pi x / d)) & \text { if }|x|<d \text { and }|y|<a / 2 \\
0 & \text { otherwise }
\end{array}\right.
$$

This was done for $d=20,40, \ldots, 100 \mathrm{~nm}$ and $\sigma=1,2, \ldots, 30$ $\mathrm{nm}$, with a pixel size of $1 \mathrm{~nm}$ and number of signal photos $n_{\mathrm{ph}}=500$. For each $d$ and $\sigma, 400$ simulations were carried out for a density of localizations $\rho=2 \times 10^{4} \mu \mathrm{m}^{-2}$. The value of $\rho$ for which the resolution was calculated was varied in these simulations by taking $2,4, \ldots 100 \%$ of the simulated localizations at $\rho=$ $2 \times 10^{4} \mu \mathrm{m}^{-2}$. However, the resolution could not be obtained by calculating where the FRC curve falls below the threshold because the FRC is not approximately monotonically decreasing for this object. Instead, contour lines in the $\rho \sigma$ plane were generated for each $d$ where $\operatorname{FRC}(1 / d)=1 / 7$. These contour lines are equivalent to lines of constant resolution $R=d$.

Figure 1c. The result in Figure 1c was obtained in a similar way as the result in Figure 1b. However, in the simulations for Figure 1b, $\rho$ and $\sigma$ are used as independent variables, whereas for Figure 1c, $\rho$ is determined by both $T_{\text {total }}$ and $\sigma: \sigma=$ $\sigma_{0} / \sqrt{ }\left(\phi_{\text {ph }} T_{\text {frame }}\right)$ and $\rho=M_{\text {sim }} T_{\text {total }} / T_{\text {frame. }}$. Here $\sigma_{0}=90 \mathrm{~nm}, \phi_{\mathrm{ph}}=$ $5.0 \times 10^{4} \mathrm{~s}^{-1}$ is the number of collected photons per emitter per unit time and $M_{\text {sim }}=0.2 \mu \mathrm{m}^{-2}$ is the number of simultaneously active emitters ${ }^{43}$. Localized emitter localizations were simulated for this figure 100 times for $T_{\text {total }}=30 \mathrm{~min}, T_{\text {frame }}=10^{-3}, 10^{-2.8}, \ldots$, $10^{-1} \mathrm{~s}$ and $d=10,15, \ldots, 60 \mathrm{~nm}$. The FRC curve was calculated by taking the localizations up to 1, 5, 10, 20 and 30 min out of these sets of localizations of $30 \mathrm{~min}$ to vary $T_{\text {total }}$. Lines of constant measurement time $T_{\text {total }}$ were then calculated by taking the contour lines in the $\sigma R$ plane where $F R C(q=1 / R)=1 / 7$.

Further information on the simulations of FRC and FRC resolution is provided in Supplementary Note 6.

Experimental setup and methods. Figure 2a: imaging of tubulin filaments. The first samples that were used for experimental validation of the results from the simulations were tubulin structures in human epithelial cervical cancer (HeLa) cells. These cells were plated on aminosilane coverslips in Lab-Tek eight-well chambers (Nunc). Cells were fixed for $10 \mathrm{~min}$ in $4 \%$ paraformaldehyde in cytoskeleton buffer (10 mM MES, pH 6.1, $138 \mathrm{mM} \mathrm{KCl,} 2 \mathrm{mM}$ EGTA, $0.32 \mathrm{M}$ sucrose and $3 \mathrm{mM} \mathrm{MgCl}_{2}$ ) and afterward put in $50 \mathrm{mM}$ ammonium chloride in phosphate buffered saline (PBS) to quench the fixation process. Subsequently, the cells were washed three times in PBS and permeabilized (0.5\% (v/v) Triton X-100) for $2 \mathrm{~min}$ with $0.2 \%$ fish-skin gelatin added to reduce nonspecific binding. Cells were then washed three times in PBS again and 
subsequently labeled with anti- $\beta$-tubulin antibodies (9F3 rabbit monoclonal) conjugated to Alexa Fluor 647 dye molecules (Cell Signaling Technology) in PBS at a concentration of approximately $1.0 \mu \mathrm{g} / \mathrm{ml}$ in the presence of $0.2 \%$ fish-skin gelatin, after which they were washed thrice in PBS. Before imaging the cells, a sparse dilution of $1: 10^{5}$ of fluorescent beads $(0.1 \mu \mathrm{m}$ TetraSpeck microspheres, Invitrogen) was put in solution for $3 \mathrm{~min}$ to enable drift correction. Next, the cells were immersed in oxygen-scavenging buffer solution consisting mainly of glucose oxidase and catalase in PBS in the presence of glucose and $80 \mathrm{mM}$ mercaptoethylamine (MEA) as a reducing agent.

Imaging of the samples was carried out in an epifluorescence microscope setup. This setup consisted of the following components: an inverted microscope (IX71, Olympus), a 1.45-NA TIRF objective (U-APO 150× NA 1.45, Olympus), a 635-nm diode laser (Radius 635, Coherent), a 561-nm diode-pumped solid state laser (CrystaLaser) and an EMCCD camera (iXon 897, Andor) with EM gain set to 25. The epifluorescence filter setup consisted of a dichroic mirror (650 nm, Semrock) and an emission filter (692/40, Semrock). The samples were mounted in a 3D piezo stage (Nano-LPS100, Mad City Labs). Images were taken in a TIRF configuration at 20 frames per second for 14,000 frames, giving a total measurement time of about $12 \mathrm{~min}$. Drift correction was accomplished by moving the field of view to a preselected fluorescent bead and imaging it with the 561-nm laser after every 1,000 frames of acquisition with the 635-nm laser on. Position estimates of the beads were then used to move the sample back to its initial position at the beginning of the experiment.

Figure 2j: imaging of actin filaments. HeLa cells were cultured on \#1.5 coverslips. After $24 \mathrm{~h}$ cells were washed briefly with PBS and fixed in two steps: a first incubation step in $0.3 \%$ glutaraldehyde $+0.25 \%$ Triton in cytoskeleton buffer (10 mM MES, pH 6.1, $150 \mathrm{mM} \mathrm{NaCl}, 5 \mathrm{mM}$ EGTA, $5 \mathrm{mM}$ glucose and $5 \mathrm{mM} \mathrm{MgCl}_{2}$ ) for $2 \mathrm{~min}$ at room temperature and a second step with $0.5 \%$ glutaraldehyde in the same buffer for $10 \mathrm{~min}$ at room temperature. The sample was treated with $0.1 \% \mathrm{NaBH}_{4}$ in PBS (freshly prepared) for $7 \mathrm{~min}$ at room temperature to reduce background fluorescence ${ }^{44}$. Samples were extensively washed with PBS and blocked with 5\% BSA for $30 \mathrm{~min}$ at room temperature. Staining with phalloidin (Invitrogen) diluted in 5\% BSA in PBS to a final concentration of $0.25 \mathrm{M}$ was done overnight at a temperature of $4{ }^{\circ} \mathrm{C}$. Cells were washed using first $0.1 \%$ Tween-20 in PBS and then PBS. Imaging of the samples was carried out on a Leica SR-GSD microscope. This setup consisted of the following components: an inverted microscope (DMI6000 B, Leica Microsystems GmbH), a 1.47-NA TIRF objective (HCX PL APO 100× NA 1.47), a tube lens providing an extra factor of $1.6 \times$ in magnification, a $532-\mathrm{nm}$ fiber laser (2RU-VFL-P-1000-532-B1R, MPB communications), a 642-nm fiber laser (2RU-VFL-P-1000-642-B1R, MPB Communications) and an EMCCD camera (iXon DU-897, Andor) with an effective EM gain of 50.6. The epifluorescence filter cube (642HP-T) for imaging with the 642-nm laser consisted of an excitation filter (zet405/642x), a dichroic mirror (t405/642rpc) and emission filters (et710 100lp and ET650LP). Images were taken in TIRF mode at 100 frames per second for 50,000 time frames, giving a total measurement time of about $8 \mathrm{~min}$. The epifluorescence filter cube (532HP-T) for imaging with the 532-nm laser consisted of an excitation filter (zet405/532x), a dichroic mirror (t405/532rpc) and emission filters (et600/100m and ET550LP).
Localization and image rendering algorithms (Fig. 2). The recorded movies were processed by estimating the emitters' positions, as well as the Cramer-Rao lower bounds (CRLBs) for those events, using a fast algorithm ${ }^{24}$ on a Quadro 5000 GPU (NVidia). The method for finding candidate regions of interest for position estimation has been documented in the literature ${ }^{38}$. Because the fitting algorithm is expected to perform close to the CRLB for each fit, these CRLBs were taken as estimates of the localization precision of the fits. The resulting events were filtered to reduce the number of false positive localizations. The parameters used for filtering were the estimated number of signal photons $n_{\mathrm{ph}}$ of the event (at least 250), the estimated localization precision $\sigma$ (at most $30 \mathrm{~nm}$ for Fig. 2a and $35 \mathrm{~nm}$ for Fig. 2j), the s.d. of the Gaussian PSF model $\sigma_{\mathrm{PSF}}(101-161 \mathrm{~nm}$ for Fig. 2a and 100-150 nm for Fig. $2 \mathbf{j}$ ) and the information divergence between the PSF model and the data in the fitted regions of interest (at least -120). Figure 2 shows a comparison between the maximum-likelihood estimation algorithm, the least-squares estimation and a centroid estimation scheme for the same localization events ${ }^{45,46}$. This means that the centroids were estimated for the same regions of interest (ROI) in the raw data that contributed to the localizations in the maximum-likelihood image. For this estimation, the background was subtracted from the ROI image, negative pixel values were set to 0 and the center of mass was subsequently calculated. The background value is the average of 24 edge pixels: the 32 edge pixels of the $9 \times 9$ pixel ROI excluding the four highest and four lowest values. Least-squares fitting was done by minimizing the mean square error between the observed ROI images and a Gaussian spot (integrated over the finite pixels) characterized by the emitter position, spot width, signal photon count and a constant background. The mean square error function was minimized using a standard Levenberg-Marquardt optimization routine programmed in Matlab.

The filtered localizations in Figure $\mathbf{2} \mathbf{j}$ were corrected for stage drift using a frame-by-frame cross-correlation algorithm ${ }^{25}$. Time series were split into $M$ (typically $M=20$ ) equal parts. For each of these parts, a super-resolution image was made by binning the localizations into bins, typically $10 \mathrm{~nm}$ in size. Subsequently, the displacement of each image with respect to the first image was calculated, and from this displacement the drift was calculated for the time points at the boundaries of each time block. Finally, the average drift per time block was computed from the two drift estimates at the boundaries of those time blocks, and these were integrated to come to an estimate of the sample's trajectory over time, which was subtracted from the estimated fluorophore locations.

The $N_{\text {raw }}$ drift-corrected localizations were condensed into $N<N_{\text {raw }}$ localization events by grouping spatially nearby localizations that are less than $\Delta$ image frames apart into single localization events, where $\Delta=5$ for the data in Figure 2. 'Nearby' is defined here as having a distance less than three times the sum of the localization uncertainty of the two to-be-merged localization events. For the single grouped localization, the position was taken to be equal to the weighted average of the localizations with the inverse of the variances as weights. Also, for each grouped localization the sum of the photon counts and background photon counts of the single localizations were stored, and the estimated variance of the grouped localization was taken to be the harmonic mean of the single localizations' variances. This procedure 
improves the localization uncertainty, as the average number of photons per localization event now scales with the fluorescence on-time $\tau_{\text {on }}$ rather than with the frame time $T_{\text {frame }}$.

Figure 3: two-color imaging of tubulin filaments. Green monkey kidney BS-C-1 cells were fixed with formaldehyde (3\%)+ glutaraldehyde $(0.1 \%)$ at room temperature in PBS for $10 \mathrm{~min}$. The fixing step was followed by quenching with sodium borohydride $(0.1 \%)$ in PBS for $7 \mathrm{~min}$. The fixed sample was permeabilized in blocking buffer (3\% BSA, 0.5\% Triton X-100 in PBS) for $10 \mathrm{~min}$ and stained with primary antibodies for $30 \mathrm{~min}$ in blocking buffer. The sample was rinsed with washing buffer (0.2\% BSA, $0.1 \%$ Triton X-100 in PBS) three times for $10 \mathrm{~min}$ each. Secondary antibodies were added to the sample (diluted in blocking buffer) and left for $30 \mathrm{~min}$ at room temperature. The sample was then washed three times for 10 min each with washing buffer. The sample was postfixed for $10 \mathrm{~min}$ at room temperature with formaldehyde $(3 \%)+$ glutaraldehyde $(0.1 \%)$ and then stored in PBS at $4{ }^{\circ} \mathrm{C}$ before imaging. For two-color imaging of microtubules labeled with both Alexa Fluor 647 and Alexa Fluor 750, the primary antibody was rat anti-tubulin (Abcam ab6160, 1:100 dilution). The secondary antibody was donkey anti-rat (Jackson ImmunoResearch, 712-005-153). Two separate labeled samples of secondary antibodies were prepared: one labeled with both Cy2 and Alexa Fluor 647 and the other labeled with Cy2 and Alexa Fluor 750, as described previously ${ }^{26}$. These two samples of secondary antibody were mixed in equal portions and used for labeling at a concentration of $\sim 2.5 \mu \mathrm{g} / \mathrm{ml}$. The microscope setup used for localization imaging has been described in detail previously ${ }^{47}$. Briefly, an inverted fluorescence microscope (Olympus IX71) was equipped with a $100 \times$ oil-immersion objective lens (Olympus, UPLANSAPO100XO) and an EMCCD camera (Andor DU-897) that enabled efficient detection of single fluorophores. A custom-built focus lock system was used to maintain sample focus during all measurements. For imaging, photoswitchable Alexa Fluor 647 or Alexa Fluor 750 was excited using 642-nm light or 752-nm light, respectively. Laser light at $642 \mathrm{~nm}$ was generated using a fiber laser (MPB Communications, 2RU-VFL-P- 1500-642), and laser light at $752 \mathrm{~nm}$ was generated using a Krypton gas laser (Coherent, Innova I300C). Additionally, the microscope was capable of laser illumination at $488 \mathrm{~nm}$, generated using an Argon-Krypton laser (Coherent, Innova I-70), and $561 \mathrm{~nm}$, generated using a solidstate laser (Cobalt, Jive). For detection of Alexa Fluor 647 fluorescence, a dichroic mirror (Chroma, Z660DCXRU) was used to split excitation light from emitted fluorescence, and a bandpass emission filter (Chroma, ET700/75) was used to filter the emitted signal. A dichroic mirror (Chroma, Q770DCXR) and a band-pass emission filter (Chroma, HQ800/60) were also used for fluorescence detection of Alexa Fluor 750. The data were reconstructed from 138,749 frames at 60 frames per second, giving a total measurement time of about $39 \mathrm{~min}$ (Alexa Fluor 647), and from 30,087 frames at 20 frames per second, giving a total measurement time of about 25 min (Alexa Fluor 750).

Imaging of tubulin labeled with both Alexa Fluor 647 and Alexa Fluor 750 was carried out in two sequential steps. First, a data set was obtained for the Alexa Fluor 750 channel, followed by a second data set for the Alexa Fluor 647 channel. Fiducial markers were used to register the two images, creating the final two-color image $^{47}$. Fluorescent beads (Invitrogen, F8810) were bound to the sample and used as fiducial markers for drift correction and image registration. Prior to imaging, the sample was incubated with a solution of beads ( $2 \%$ solids stock solution diluted 1:50,000 in PBS), which were allowed to bind to the sample for $1 \mathrm{~min}$. The sample was then rinsed and incubated with PBS $+50 \mathrm{mM} \mathrm{MgCl}_{2}$, which caused the beads to stick to the surface of the coverglass. The buffer was then exchanged to imaging buffer, and the data set was collected.

The imaging medium consisted of a $\mathrm{pH}$ buffer with an enzymatic oxygen-scavenging system consisting of glucose, glucose oxidase, catalase to reduce photobleaching and a thiol to facilitate photoswitching. The specific composition of the imaging buffer was Tris $(50 \mathrm{mM}, \mathrm{pH} 8.0), \mathrm{NaCl}(10 \mathrm{mM})$, glucose $(10 \% \mathrm{w} / \mathrm{v})$, $\beta$-mercaptoethanol (143 mM) and the enzymatic oxygen scavenging system $(1 \% \mathrm{v} / \mathrm{v})$. The enzymatic oxygen-scavenging system stock solution (GLOX) was prepared by mixing glucose oxidase powder (10 mg, Sigma, G2133) with catalase (50 $\mu$ l, Sigma, C100) in PBS $(200 \mu \mathrm{l})$ and centrifuging the mixture at 13,000 r.p.m. for $1 \mathrm{~min}$. The data analysis used for localization microscopy and the nanoscale image-registration procedure, based on the positions of the fiducial markers, has been described in detail previously ${ }^{26}$.

Figure 4: 3D imaging of tubulin filaments. Swiss 3T3 cells were plated in eight-well chambers (Lab-Tek II, Nunc) overnight in standard DMEM phenol-free medium. Samples were washed twice with room-temperature PBS and then fixed with $4 \%$ PFA in cytoskeleton buffer (10 mM MES, $138 \mathrm{mM} \mathrm{KCl,} 3 \mathrm{mM} \mathrm{MgCl}$, $2 \mathrm{mM}$ EGTA, $0.32 \mathrm{M}$ sucrose) for $30 \mathrm{~min}$ at room temperature. The sample was then incubated twice for $5 \mathrm{~min}$ in $10 \mathrm{mM}$ Tris in PBS. The sample was permeabilized with a $1 \%$ BSA, $0.1 \%$ Triton solution in PBS for 15 min. During the permeabilization the primary antibodies were added to an aliquot of the previously mentioned blocking/permeabilizing buffer at a concentration of $12 \mu \mathrm{g} / \mathrm{ml}$. After permeabilization, the sample was incubated with the primary antibody (Sigma T8328 anti-B tubulin) at $12 \mu \mathrm{g} / \mathrm{ml}$ in permeabilization buffer for $1 \mathrm{~h}$ at room temperature on an orbital shaker operating at a slow speed. The sample was then washed three times for 5 min each with $1 \%$ BSA, $0.1 \%$ Triton solution. Secondary antibody labeling was performed using an anti-mouse antibody (Jackson ImmunoResearch, 715-005-150 anti-mouse IgG) labeled with an average of two Alexa Fluor 647 dyes per protein. Labeling was performed at a concentration of $15 \mu \mathrm{g} / \mathrm{ml}$ in permeabilization buffer for $30 \mathrm{~min}$ at room temperature on an orbital shaker. The sample was again washed three times for $5 \mathrm{~min}$ in PBS, postfixed for $10 \mathrm{~min}$ in $4 \%$ PFA and stored in $0.05 \%$ PFA in PBS solution until the time of imaging. Before imaging, samples were washed twice with $10 \mathrm{mM}$ Tris for $5 \mathrm{~min}$.

3D imaging was performed using a dual focal plane setup. To define the imaging area, we placed an adjustable slit at the primary image position of the microscope (Olympus IX71), followed by a relay lens system (focal length $F=75 \mathrm{~mm}$ and $50 \mathrm{~mm}$, respectively) to create a $1.5 \times$ magnification to a secondary image position. From there, a lens $(F=125 \mathrm{~mm})$ was used to collimate the beam, and a 50/50 beam splitter was used to split the beam into two equal-length optical paths. In one pathway an additional lens $(F=1 \mathrm{~m})$ was used to create the second focal plane, giving an approximately 330-nm defocused imaged as compared to the unaltered beam path. The two optical paths were redirected by mirrors (two in each path) so that they both pass through an imaging lens $(F=125 \mathrm{~mm})$ and image side by side 
onto the same EMCCD camera (Andor iXon 860). An emission filter (FF01-692/40-25, Semrock) was placed after the $F=125 \mathrm{~mm}$ collimation lens. Excitation light was provided by a $637-\mathrm{nm}$ diode laser (Thorlabs HL63133DG). Samples were imaged in an oxygen-scavenging buffer consisting of $10 \%(\mathrm{w} / \mathrm{v})$ glucose, $50 \mathrm{mM}$ Tris, $10 \mathrm{mM} \mathrm{NaCl}, \mathrm{pH} 8.5$, glucose oxidase, catalase and $20 \mathrm{mM}$ MEA. Excitation at $637 \mathrm{~nm}$ (Thorlabs HL63133DG) was $1 \mathrm{~kW} / \mathrm{cm}^{2} .50,000$ images were acquired at a $100-\mathrm{Hz}$ frame rate. A 405-nm laser (DL405-010-O, CrystaLaser) was used to recover Alexa Fluor 647 from the dark state, and the power was adjusted by hand to provide control over the active-state duty cycle. Position estimations were performed using maximumlikelihood estimation with a Poisson noise model and PSF models calculated from phase-retrieved pupil functions ${ }^{48}$. Pupil functions were retrieved for each focal plane independently. The parameters position $x, y$ and $z$, intensity $I$ and background values $\mathrm{bg}_{1}$ and $\mathrm{bg}_{2}$ were estimated simultaneously using both focal planes, where $(x, y)$ positions in each plane were connected by a predetermined transform matrix, the $z$ position was connected by a plane separation that was found in the phase-retrieving process and the intensity $I$ was related with a premeasured ratio factor.
Further information on the experimental setup for the supplementary figures is provided in Supplementary Note 7.

41. Tukey, J.W. An introduction to the calculations of numerical spectrum analysis. in Spectral Analysis of Time Series (ed. Harris, B.) 25-46 (Wiley, New York, 1967).

42. Cleveland, W.S. Robust locally weighted regression and smoothing scatterplots. J. Am. Stat. Assoc. 74, 829-836 (1979).

43. Wolter, S., Endesfelder, U., van de Linde, S., Heilemann, M. \& Sauer, M. Measuring localization performance of super-resolution algorithms on very active samples. Opt. Express 19, 7020-7033 (2011).

44. Xu, K., Babcock, H.P. \& Zhuang, X. Dual-objective STORM reveals threedimensional filament organization in the actin cytoskeleton. Nat. Methods 9, 185-188 (2012).

45. Ghosh, R.N. \& Webb, W.W. Automated detection and tracking of individual and clustered cell surface low density lipoprotein receptor molecules. Biophys. J. 66, 1301-1318 (1994).

46. Lee, G.M., Ishihara, A. \& Jacobson, K.A. Direct observation of Brownian motion of lipids in a membrane. Proc. Natl. Acad. Sci. USA 88, 6274-6278 (1991).

47. Bates, M., Jones, S.A. \& Zhuang, X. Stochastic optical reconstruction microscopy: a method for superresolution fluorescence imaging. in Imaging: A Laboratory Manual (ed. Yuste, R.) Ch. 35, 547-576 (Cold Spring Harbor Laboratory Press, 2011).

48. Hanser, B.M., Gustafsson, M.G., Agard, D.A. \& Sedat, J.W. Phase-retrieved pupil functions in wide-field fluorescence microscopy. J. Microsc. 216, 32-48 (2004). 